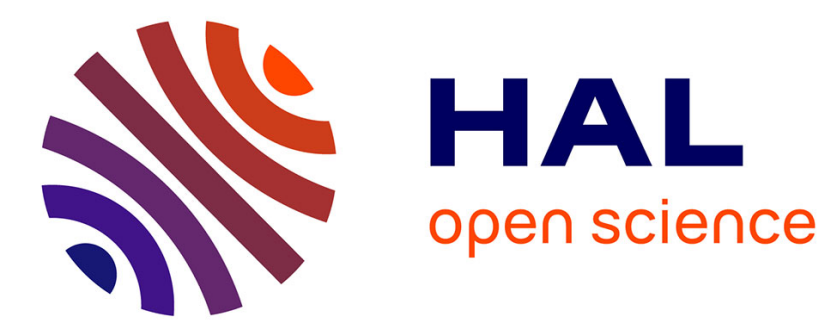

\title{
Photoelectron microscopy from magnetic materials
}

\author{
T. Kachel, K. Holldack, W. Guda, M. Neuber, C. Wilde
}

\section{To cite this version:}

T. Kachel, K. Holldack, W. Guda, M. Neuber, C. Wilde. Photoelectron microscopy from magnetic materials. Journal de Physique IV Proceedings, 1994, 04 (C9), pp.C9-439-C9-444. 10.1051/jp4:1994972 . jpa-00253534

\section{HAL Id: jpa-00253534 https://hal.science/jpa-00253534}

Submitted on 1 Jan 1994

HAL is a multi-disciplinary open access archive for the deposit and dissemination of scientific research documents, whether they are published or not. The documents may come from teaching and research institutions in France or abroad, or from public or private research centers.
L'archive ouverte pluridisciplinaire HAL, est destinée au dépôt et à la diffusion de documents scientifiques de niveau recherche, publiés ou non, émanant des établissements d'enseignement et de recherche français ou étrangers, des laboratoires publics ou privés. 


\title{
Photoelectron microscopy from magnetic materials
}

\author{
T. Kachel, K. Holldack, W. Gudat, M. Neuber* and C. Wilde* \\ BESSY, Berlin, Germany \\ * Inst. Appl. Phys. Chem., University of Heidelberg, Heidelberg, Germany
}

Synchrotron radiation based photoelectron microscopy is discussed. We show that the spectromicroscope ESCASCOPE together with circularly polarized VUV radiation can be used to measure magnetic domains with $10 \mu \mathrm{m}$ resolution. The high element specifity of the setup allows to detect domains in monoatomic overlayers and to distinguish them from the substrate domain structure.

\section{Introduction}

Photoelectron spectroscopy and related methods have played a major role in the understanding of surface properties during the last three decades. These techniques are sensitive probes of the electronic properties related to chemistry, structures and band structures of solids and their surfaces over a wide variety of materials like semiconductors, metals and magnetic materials. The field gained strongly from the increased access to synchrotron radiation sources. On this basis scientists found improved situations concerning the photon flux rates and the tunability of the soft X-ray radiation which are strictly required for many sorts of experiments. The surface sensitivity of those kinds of experiments is inherently very high and this fact might be understood as a very high spatial resolution perpendicular to the surface plane. But there has been also a steady improvement in the field of microanalysis, i.e. in the lateral spatial resolution, for both microprobes and microscopes along with the technical advances in soft $\mathrm{X}$-ray sources and monochromator design.

Microscopic methods using soft $\mathrm{X}$-ray radiation cover quite a number of different techniques, as for example the use of zone plates in photon transmission experiments or photoemission microscopes in the scanning and in the parallel imaging mode. Big efforts have been made in the field of transmission microscopy particularly because of the idea of working with wave lengths smaller than in the optical range to lower the constraints of the diffraction limits. Very successful experiments have been reported in the past, showing e.g. microimages of biological matter with $30 \mathrm{~nm}$ resolution. ${ }^{1}$ In photoelectron microscopy UV or soft $\mathrm{X}$-ray radiation is required to exceed the work function barrier of solids. With photoelectron microscopy it has been shown possible to acquire photoelectron images of surfaces with a spatial resolution down to below $10 \mathrm{~nm} .{ }^{2}$ When using standard low energy UV emitters like e.g. Hg pressure lamps it is mainly the work function change in spatial distributions of different materials or of edges in surface layers that is reflected in the micro-images. Also in this field the use of tunable synchrotron radiation is of strong interest as 
it allows to sweep the photon energies through absorption edges of particular elements and, thus, to identify their location on sample surfaces. Consequently, this kind of work should therefore be understood as mainly chemical microscopy.

Another step foreward in photoemission microscopy can be made when taking advantage not only of the high flux, high brilliance and tunability but also of its unique behaviour in the light polarization of synchrotron radiation. During the past few years there has been an enormous effort and success in experimental ${ }^{3}$ and theoretical ${ }^{4}$ description of the magnetic circular dichroism (MCD) effect. MCD appears as differences in absorption and emission probabilities depending on the relative alignments of the average photon spin in circularly polarized soft X-rays and magnetization vectors in magnetic materials.

\section{Instrumental Considerations}

The study and the application of MCD requires photon sources emitting soft X-rays of high circular polarization over a large photon energy range and monochromator beamlines transmitting the radiation without strong influence on the polarization. Normal incidence monochromators using off-plane bending magnet circularly polarized UV radiation are in operation already since more than a decade. Because of the decreasing reflectance of optical elements in normal incidence at higer photon energies these systems are limited to an energy range below about $30 \mathrm{eV}$. Hence, the core levels of magnetic materials like the 3d transition metals and those core levels of the lanthanides that give rise to the prominent absorption edges are not accessible this way.

Recently there have been also substantial advances in building both, sources and monochromators for circularly polarized soft $\mathrm{X}$-rays for an extended energy range. Off-plane radiation from bending magnets, radiation from crossed, helical or asymmetric undulators and wigglers are possible sources. Also $\lambda / 4$ - plate phase shifters like, depending on the photon energy, transmission multilayers or single crystal structures are discussed as such. The related monochromators must be equipped with grazing incidence optical elements only. These requirements can be fulfilled and therefore standard photoelectron microscopy can be extended to magnetic microscopy. In our work we employed the so called SX 700 III beamline at BESSY. ${ }^{5}$ The SX 700 is a plane grating monochromator optimized for the spectral range of about $30-1400 \mathrm{eV}$. The monochromator is equipped with a special double entrance mirror system, one transmitting $\sigma^{-}$and $\pi$, the other transmitting $\sigma^{+}$polarized light. With an aperture system in front of the double mirror it is then possible to tune the bending magnet radiation take-off angle with respect to the storage ring plane. Hence, one can continuously change also the degree of circular polarization. Fig. 1 reproduces results from the monochromator characterization. It shows photon flux curves for 0 ( $\pi$ polarization) and 0.6 $\operatorname{mrad}\left(\sigma^{ \pm}\right.$polarization) take-off angle that we have used for our experiments. In the same figure we have included the calculated polarization dependence at $0.6 \mathrm{mrad}$. Estimates of the circular polarization from experimetal results are in good agreement with the calculated behaviour.

The photoelectron microscope that we used for our experiments is the commercial parallel imaging Fisons ESCASCOPE. ${ }^{6}$ Fig. 2 shows a schematic drawing of the ESCASCOPE's analyzer and lens system. The instrument is equipped with a hemispherical electrostatic energy analyzer offering an energy resolution of about $2 \%$ of the selected pass energy. In the imaging mode we have chosen a constant retard ratio of 4 . A very special lens system serves for the conservation of the spatial information that the photoelectrons carry on their pass through the energy analyzer. Electrons are multiplied by a double channelplate system and projected onto a fluorescent screen where the signal is taken off and digitized by a CCD camera system. The microscope has a spatial resolution of $10 \mu \mathrm{m}$ and fields of view can be selected between minimum $50 \mu \mathrm{m}$ diameter and $500 \mathrm{x}$ 


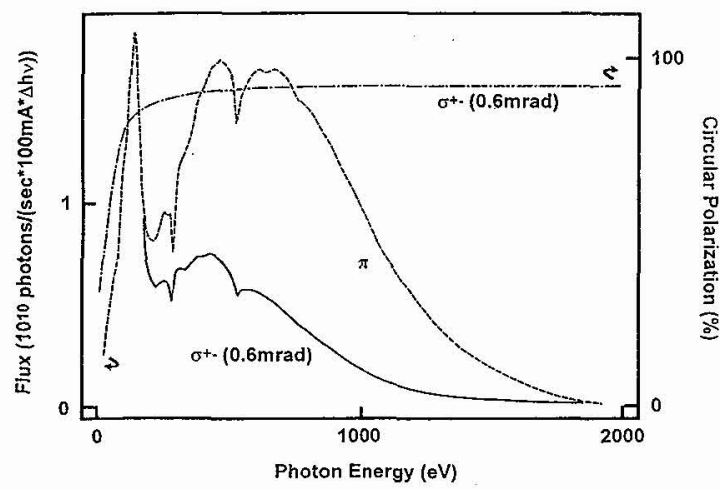

Figure 1: Photon flux curves of the SX 700 III monochromator at BESSY. Dashed line: in-plane ( $\pi$ - polarized) emission, full line: $0.6 \mathrm{mrad}$ off-plane $\left(\sigma^{+-}\right.$polarized) emission, dashed-dotted line: polarization of the 0.6 mrad off-plane emission. (data extracted from ref. 5)

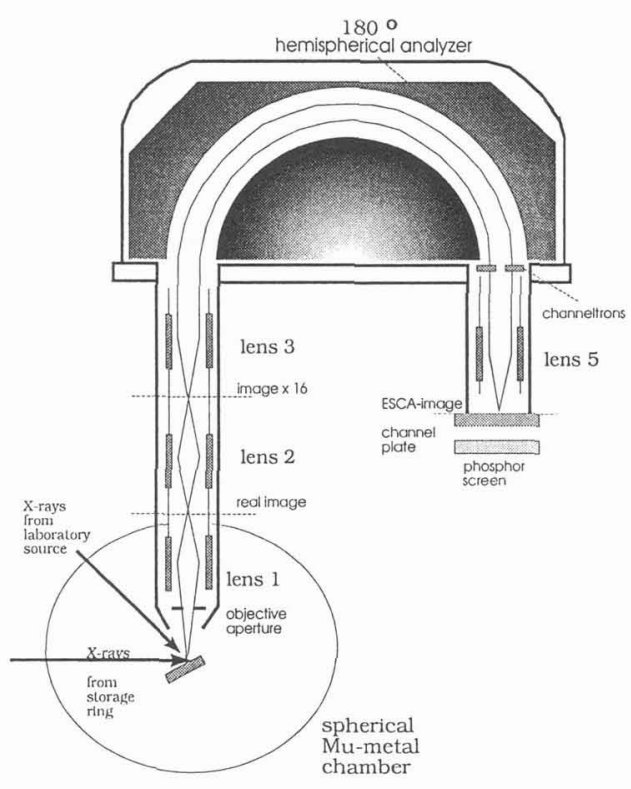

Figure 2: Scheme of the ESCASCOPEs electron energy analyzer and lens system.

$800 \mu \mathrm{m}$ maximum. The instrument can be easily operated in a regular photoelectron spectrometer mode, when reading out not the CCD camera but a set of 6 channeltrons. Furthermore, it is possible to use the spectrometer with a reduced field of view, i.e. the instrument can work as a photoemission microprobe.

\section{Results and Discussion}

\section{3a. Magnetic Imaging from Fe}

In principle, any sort of MCD effect in photoemission from core levels and valence bands, resonant photoemission or Auger electron emission can be used as the basic effect for magnetic photoelectron microscopy. Electron counting rates can differ for varying alignment of photon spin and magnetization direction at a given photon energy, electron binding- or kinetic energy and perhaps the emission angle. In every case of such a variation magnetic domain structures will lead to spatial distributions of the electron emission intensities. Practically, however, it is important to achieve optimized parameters for high flux and polarization of the monochromator and high MCD asymmetry and photoabsorption cross sections in the material to be studied. Fig. 3 shows the basic effect for studying magnetic domains on Fe following the lines of Stöhr et al. ${ }^{7}$ who observed an analogous behaviour for Co. In the figure we display NEXAFS spectra from the Fe 2p edge taken with $\sigma^{-}$radiation of about $85 \%$ circular polarization which are similar to the results of Schneider et al. ${ }^{8}$. For this spectrum we collected electrons with a kinetic energy of $703 \mathrm{eV}$ which corresponds to the maximum of the Fe LVV Auger emission. The dotted curve is taken for nearly (angle of incidence $\theta=25^{\circ}$ ) antiparallel, the full line curve for nearly parallel alignment of photon spin and magnetization vector. One mainly observes very strong intensity changes close to the $L_{3}$ and the $\mathrm{L}_{2}$ absorption edges.

The MCD asymmetries at these photon energies can be successfully used to measure magnetic 


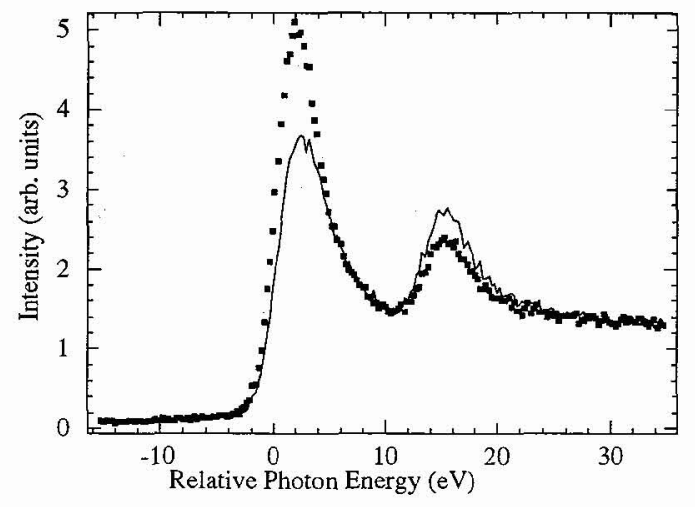

Figure 3: NEXAFS spectra from the $\mathrm{Fe} \mathrm{L}_{3}$ and $L_{2}$ edges taken with about $85 \%$ circularly polarized soft $\mathrm{X}$-rays. Electron kinetic energy $\mathrm{E}_{k i n}=703 \mathrm{eV}$. Full line: parallel, squares: antiparallel alignment of photon spin and magnetization. Angle of light incidence: $25^{\circ}$. (redrawn from ref. 9)

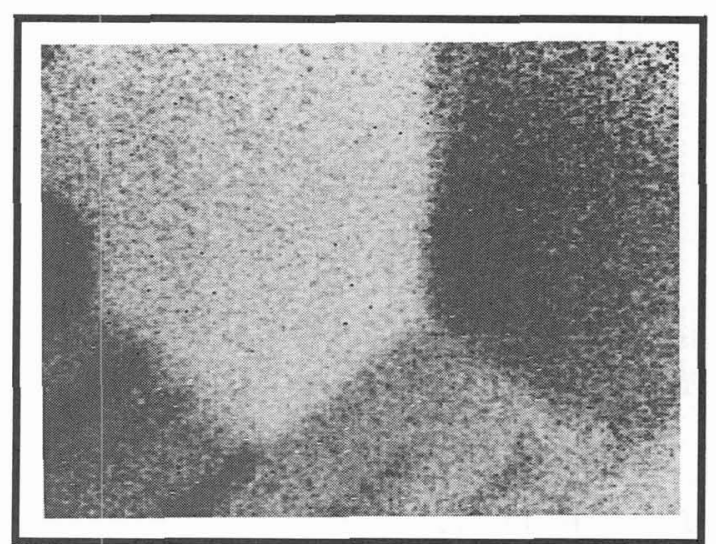

Figure 4: Micrograph of magnetic domains on an Fe (100) crystal with dislocations. ESCASCOPE data (asymmetry image). Spatial resolution: $10 \mu \mathrm{m}$, field of view: $650 \times 500 \mu \mathrm{m}$.

domain images. In order to do so we work with pixel-by-pixel asymmetry images. Two micrographs are taken from the same sample position with photon energies corresponding to the $L_{3}$ and the $L_{2}$ absorption edges, respectively, hence with reversed magnetic contrast. Pixel-by-pixel differences of the images are obtained numerically and the result is normalized to their sum, i.e. $\left(\mathrm{I}^{p}-\mathrm{I}^{a}\right) /\left(\mathrm{I}^{p}+\mathrm{I}^{a}\right)$, with $\mathrm{I}^{p}$ the image for parallel and $\mathrm{I}^{a}$ the image for antiparallel photon spin and magnetization. Compared to the raw data the resulting asymmetry images have improved magnetic contrast, because other image contrast as e.g. due to structure is canceled. Also an intensity envelope function in the raw data arising from non-homogeneous sample illumination by the $\mathrm{X}$-rays is compensated for. In fig. 4 we present such an asymmetry domain image. In the upper part of the image we find two large antiparallel domains separated by a domain wall. Field loops are closed in the typical way by a domain with a magnetization vector tilted by $90^{\circ}$. The domain structure is, however, overlapped by magnetic irregularities, particularly in the lower and left part of the image and at the top part of the big vertical domain wall. These irregularities are due to dislocations in the crystal structure of the sample we have used. This micrograph gives an example of the quality with which one can obtain magnetic domain images even down to possible fine structure.

\section{3b. Magnetic Imaging from Rare Earth Monolayers}

A more challenging goal besides measuring magnetic domains on pure $3 \mathrm{~d}$ metals is, to our understanding, the exploitation of the great advantages of our instrumental setup. Those are, particularly, the high element specifity and surface sensitivity. To demonstrate the strength of the method we have taken micro-images of magnetic domains in monoatomic overlayers of Gd and $\mathrm{Tb}$ on $\mathrm{Fe}(100) .^{9}$ The overlayers have been prepared in situ by electron beam evaporation. From earlier spin resolved photoelectron spectroscopy and LEED experiments we have already a detailed knowledge of the structural and magnetic behaviour of these systems. ${ }^{10}$ It is known that the two elements grow on $\mathrm{Fe}(100)$ in a layer by layer mode but not epitaxially. The first rare earth layer is magnetically ordered at room temperature and it couples antiferromagnetically to the Fe substrate magnetization. An increase of the overlayer thickness leads to an attenuation of the 


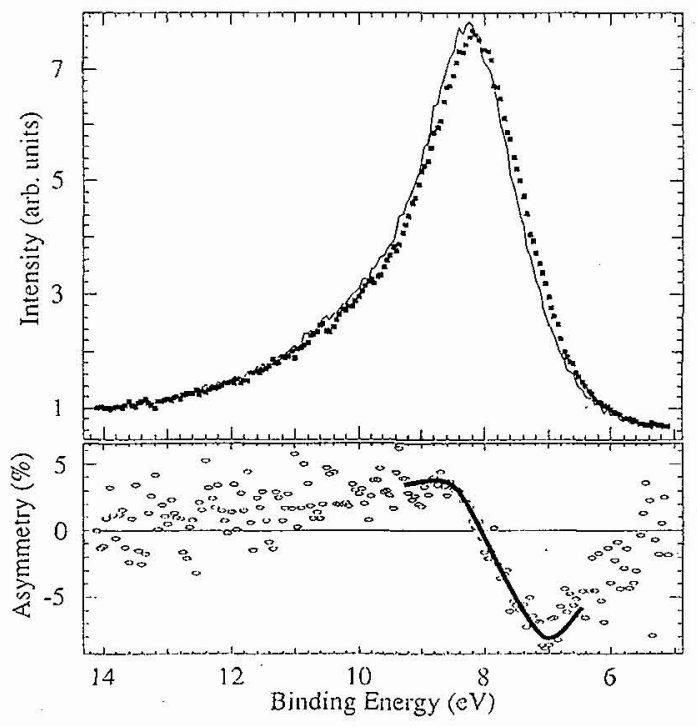

Figure 5: Top: energy distribution curves of the Gd $4 \mathrm{f}$ photoemission from $1 \mathrm{ML} \mathrm{Gd} / \mathrm{Fe}$ (100) taken with about $65 \%$ circularly polarized soft $\mathrm{X}$-rays at $\mathrm{h} \nu=152 \mathrm{eV}$ (maximum of the $4 \mathrm{~d}$ - $4 \mathrm{f}$ giant resonant excitation). Full line: parallel, squares: antiparallel alignment of photon spin and magnetization vectors. Angle of light incidence: 25. Bottom: corresponding asymmetry curve: $\mathrm{A}=$ $\left(\mathrm{I}^{p}-\mathrm{I}^{a}\right) /\left(\mathrm{I}^{p}+\mathrm{I}^{a}\right)$. (from ref. 9 )

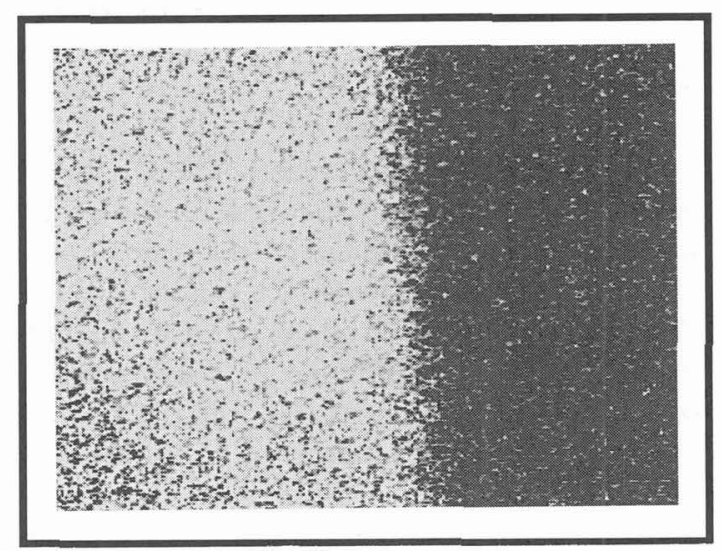

Figure 6: Micrograph of two antiparallel magnetic domains in a monoatomic $\mathrm{Tb}$ layer on an $\mathrm{Fe}(100)$ crystal. Spatial resolution: $10 \mu \mathrm{m}$, field of view: $650 \times 500 \mu \mathrm{m}$.

spin polarization and MCD signal and at about 4 - $5 \mathrm{ML}$ the magnetic signal vanishes completely. Furthermore, very recently Starke et al. ${ }^{11}$ have observed strong MCD effect in photoemission from the $4 \mathrm{f}$ states of $\mathrm{Gd}$ and $\mathrm{Tb}$ which can be used for the magnetic micro-imaging.

In order to explain the basic effect employed in the magnetic microscopy we present in fig. 5 photoelectron spectroscopy data from the Gd 4 f emission region of $1 \mathrm{ML} \mathrm{Gd} \mathrm{/} \mathrm{Fe} \mathrm{(100).} \mathrm{The} \mathrm{spectra}$ were taken with $\sigma^{-}$polarized light of about $65 \%$ circular polarization at a photon energy $h \nu=152$ $\mathrm{eV}$, corresponding to the maximum of the Gd giant $4 \mathrm{~d}-4 \mathrm{f}$ resonant emission. This choice enhances the overlayer contribution to the total photoemission signal. The full line represents the results for parallel, the dotted line those for antiparallel alignment of photon spin and magnetization. The lower part of the figure shows the corresponding intensity asymmetry curve. It is seen that the MCD asymmetry changes sign over the Gd $4 f$ peak with a maximum of about $4 \%$ at higher and a minimum of about $-8 \%$ at lower binding energy. Also in the $\mathrm{Tb} 4 \mathrm{f}$ photoemission from 1 ML Tb / Fe (100) we have found strong MCD asymmetries. This demonstrates the possibility to attain magnetic domain images of these systems in the same manner as in the case of the Fe NEXAFS spectrum. As an example we show in fig. 6 an image of two antiparallel domains separated by a domain wall in a single monolayer of $\mathrm{Tb}$ on $\mathrm{Fe}(100)$. The major advantage in this kind of microscopy is the fact that it allows a clear separation of the magnetic contributions from the different elements involved. Because of the antiferromagnetic coupling of the rare earth monolayers to the underlying $\mathrm{Fe}(100)$ one directly observes inverted magnetic contrast for the overlayers and the substrate. This inversion allows the discrimination between different magnetic 
orientations of the different elements unequivocably.

\section{Summary}

We have shown that the use of the photoelectron microscope ESCASCOPE in combination with circularly polarized soft X-rays from the SX $700 \mathrm{III}$ soft X-ray monochromator at BESSY allows to measure magnetic domain images with $10 \mu \mathrm{m}$ spatial resolution. The method is sensitive to MCD effects in photoabsortion which can be used favourably to measure domain structures in $\mathrm{Fe}$ and $\mathrm{Co}$. Because of the application of an electron energy analyzer with good energetic resolution it is also possible to separate different final states and, thus, to employ strong MCD effect in photoemission of the rare earth elements $\mathrm{Gd}$ and $\mathrm{Tb}$ for magnetic micro-imaging. Because of the high element specifity and surface sensitivity of the setup we have been able to investigate magnetic domains in monoatomic layers on a ferromagnetic substrate and to distinguish them from the substrate's domain structure which has not been shown possible by other microscopic methods so far.

\section{Acknowledgement}

We are indepted to H. Petersen, F. Schäfers and M. Willmann from BESSY for their fruitful discussions and scientific and technical support.

\section{References}

[1] Schmahl G., Rudolph D., Niemann B., Guttmann P., Thieme J., Schneider G., David C., Diehl M., Wilhein T., Optik 93, (1993) 95, and references therein.

[2] for an overview see Griffith O.H. and Engel W., Ultramicroscopy 36, (1991) 1.

[3] Schütz G., Wagner W., Wilhelm W., Kienle P., Zeller R., Frahm P., and Materlik G., Phys. Rev. Lett. 58, (1987) 737.

[4] see e.g. Thole B.T., van der Laan G., Phys. Rev. B44, (1991) 12424.

[5] Petersen H., Willman M., Schäfers F., and Gudat W., Nucl. Instr. Meth. A333, (1993) 594.

[6] Coxon P., Krizek J., Humpherson M., and Wardell I.R.M.,

J. Electron Spectrosc. Rel. Phenom. 51/52, (1990) 263.

We are indepted to Prof. Grunze, University of Heidelberg, for making the access to the experimental setup possible.

[7] Stöhr J., Wu Y., Sarmant M.G., Hermsmeier B.D., Harp G., Koranda S., Dunham D., and Tonner B.P., Science 259, (1993) 658.

[8] Schneider C.M., Meinel K., Holldack K., Oepen H.-P., Grunze M., and Kirschner J., Mat.Res. Soc. Symp. Proc. 313, (1993) 616.

[9] Kachel T., Gudat W., and Holldack K., Appl. Phys. Lett. 64, (1994) 655.

[10] Carbone C. and Kisker E., Phys. Rev. B36, (1987) 1280.

Taborelli M., Allenspach R., Boffa G., and Landolt M., Phys. Rev. Lett. 56, (1987) 2869.

Kachel T., Rochow R., Gudat W., Jungblut R., Rader O., and Carbone C., Phys. Rev. B45, (1992) 7267.

[11] Starke K., Navas E., Baumgarten L., and Kaindl G., Phys. Rev. B48, (1993) 1329. 